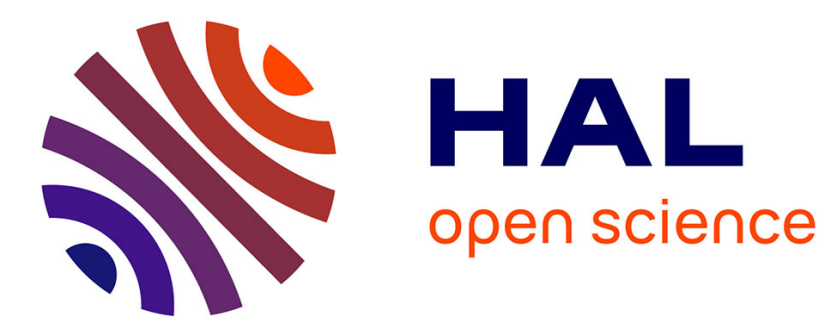

\title{
Images from the surface of asteroid Ryugu show rocks similar to carbonaceous chondrite meteorites
}

\author{
R. Jaumann, N. Schmitz, T.-M. Ho, S. Schröder, K. Otto, K. Stephan, S. \\ Elgner, K. Krohn, F. Preusker, F. Scholten, et al.
}

\section{- To cite this version:}

R. Jaumann, N. Schmitz, T.-M. Ho, S. Schröder, K. Otto, et al.. Images from the surface of asteroid Ryugu show rocks similar to carbonaceous chondrite meteorites. Science, 2019, 365 (6455), pp.817-820. 10.1126/science.aaw8627 . hal-02381801

\section{HAL Id: hal-02381801 \\ https://hal.science/hal-02381801}

Submitted on 23 Dec 2020

HAL is a multi-disciplinary open access archive for the deposit and dissemination of scientific research documents, whether they are published or not. The documents may come from teaching and research institutions in France or abroad, or from public or private research centers.
L'archive ouverte pluridisciplinaire HAL, est destinée au dépôt et à la diffusion de documents scientifiques de niveau recherche, publiés ou non, émanant des établissements d'enseignement et de recherche français ou étrangers, des laboratoires publics ou privés. 


\section{Images from the surface of asteroid Ryugu show rocks similar to carbonaceous chondrite}

\section{meteorites}

Authors: R. Jaumann ${ }^{1,2, *}$, N. Schmitz ${ }^{1}$, T.-M. Ho ${ }^{3}$, S.E. Schröder ${ }^{1}$, K.A. Otto ${ }^{1}$, K. Stephan ${ }^{1}$, S. Elgner $^{1}$, K. Krohn ${ }^{1}$, F. Preusker ${ }^{1}$, F. Scholten ${ }^{1}$, J. Biele ${ }^{4}$, S. Ulamec ${ }^{4}$, C. Krause ${ }^{4}$, S. Sugita ${ }^{5}$, K.-

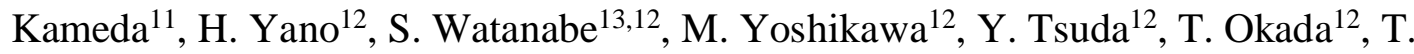

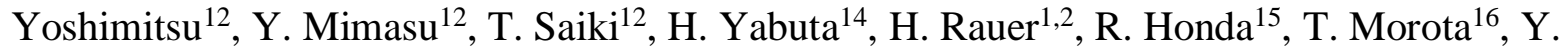

$10 \quad$ Yokota $^{12}$, T. Kouyama ${ }^{17}$

\section{Affiliations:}

${ }^{1}$ German Aerospace Center (DLR), Inst. of Planetary Research, Berlin, Germany

${ }^{2}$ Freie Univ. Berlin, Inst. of Geosciences, Berlin, Germany

${ }^{3}$ DLR, Inst. of Space Systems, Bremen, Germany

${ }^{4}$ DLR, Microgravity User Support Center, Linder Höhe, Cologne, Germany

${ }^{5}$ Dept. of Earth and Planetary Science, School of Science, University of Tokyo, Tokyo 1130033, Japan

${ }^{6}$ Univ. Côte d'Azur, Observatoire de la Côte d'Azur, Centre National de la Recherche Scientifique, Laboratoire Lagrange, Nice, France

$20 \quad{ }^{7}$ DLR, Inst. of System Dynamics and Control, Oberpfaffenhofen, Germany

${ }^{8}$ DLR, Inst. of Robotics and Mechatronics, Oberpfaffenhofen, Germany 
${ }^{9}$ Centre National d'études Spatiales, 18 Avenue E. Belin, Toulouse 31401, France

${ }^{10}$ Univ. de Paris Sud-Orsay, Institut d'Astrophysique Spatiale, Orsay, France

${ }^{11}$ Dept. of Physics, Rikkyo University, 3-34-1 Nishi-Ikebukuro, Toshima, Tokyo 171-8501, Japan

${ }^{12}$ Inst. of Space and Astronautical Science, Japan Aerospace Exploration Agency, Sagamihara, Japan

${ }^{13}$ Dept. of Earth and Planetary Sciences, Nagoya Univ. Furo-cho Chikusa-ku, Nagoya, Japan

${ }^{14}$ Dept. of Earth and Planetary Systems Science, Hiroshima University, Hiroshima, Japan

${ }^{15}$ Kochi University, Dept. of Information Science, Akebono, Kochi, Japan

${ }^{16}$ Univ. of Tokyo, Dept. of Earth and Planetary Science, Hongo, Bunkyo, Tokyo, Japan

${ }^{17}$ National Institute of Advanced Industrial Science and Technology, Aomi, Koto, Tokyo, Japan

*Correspondence to: Ralf. Jaumann@dlr.de.

Abstract: The near-earth asteroid (162173) Ryugu is a $900 \mathrm{~m}$ sized dark object supposed to contain primordial material from the solar nebula. The Mobile Asteroid Surface Scout (MASCOT) landed on Ryugu's surface on 2018 October 3. We present images from the MASCOT Camera (MASCam) taken during the descent and while on the surface. The surface is covered by decimeter to meters sized rocks, with no deposits of fine-grained material. Rocks appear either bright, with smooth faces and sharp edges, or dark, with a cauliflower-like crumbly surface. Close-up images of a rock of the latter type reveal a dark matrix with small, bright, 
spectrally different inclusions implying that it did not experience extensive aqueous alteration. The inclusions appear similar to those in carbonaceous chondrite meteorites.

One Sentence Summary: The presence of abundant multi-color, millimeter-sized inclusions in a Ryugu rock indicate strong similarities with carbonaceous chondrites.

Main text:

Japan Aerospace Exploration Agency's (JAXA) Hayabusa2 sample return mission (1), carrying the MASCOT lander, arrived at asteroid Ryugu on 2018 June 27 (2). Its initial measurements showed that that Ryugu has an equatorial radius of $502 \pm 2 \mathrm{~m}$, a bulk density of $1.19 \pm 0.02 \mathrm{~g}$ $\mathrm{cm}^{-3}$, and spectral properties typical for carbonaceous chondritic material of Cb-type (2). Ryugu's visible geometric albedo is $4.5 \pm 0.2 \%$, making it one of the darkest objects in the Solar System (3). The German Aerospace Center (DLR) developed MASCOT in cooperation with the French Centre National d'Etudes Spatiales (CNES) (4). MASCOT was released from the Hayabusa2 mothership at 01:57:20 UTC on 2018 October 3, from an altitude of $41 \mathrm{~m}$ above the surface of Ryugu (5). At 02:03:14 UTC, the lander made first contact with the surface (at contact point CP1), impacting the shadowed side of a large boulder. MASCOT then bounced backward and traveled a further $17 \mathrm{~m}$ over the surface to come to its first rest at settlement point 1 (SP1) in a local depression (Fig. 1A-C). During the six-minute descent toward the surface and the bouncing phase, the MASCam camera (6) took 20 images. In the same period, Hayabusa2's Optical Navigation Camera (ONC) $(3,7)$ recorded both the shadow of MASCOT on the surface, and the lander itself when its bright, white top was visible. These images allowed us to reconstruct the descent and bouncing trajectory (Fig.1) (5). An up-raising maneuver (4, 5), at this measurement position (MP1) (Fig. 1B) left the lander upside down with most instruments looking into the sky. A sequence of 5 images acquired during the first night on the asteroid 
shows three moving objects, whose angular velocities coincide with Ryugu's rotation period of $7.63262 \pm 0.00002$ hours (2). We identified these as Jupiter, Saturn, and the 2.0 magnitude star $\sigma$ Sgr. In the morning of the second day, MASCOT activated an internal mobility unit to reach a second location (MP2) (Fig. 1B), now properly orientated for its instruments to study the surface. Here MASCam initiated a full day/night imaging cycle, using an onboard array of LEDs to illuminate the area in front of the camera (Fig. 2). On the morning of the third day, the lander slipped about $5 \mathrm{~cm}$ sideward by executing a mini-move to enable stereo imaging for photogrammetric analysis, reaching a third location (MP3) (Fig. 1B and 2C) (5). Additional imaging over that day (Fig. 2D) enabled us to observe the surface at changing illumination and viewing geometries (5). ONC also imaged MASCOT close to the lander's end-of-mission, allowing us to constrain its final location after the $2^{\text {nd }}$ relocation to MP4 as $22.31 \pm 0.05^{\circ} \mathrm{S}$, $317.16 \pm 0.05^{\circ} \mathrm{E}(5)$. After $17 \mathrm{~h} 7 \mathrm{~min}$ of surface operations, MASCOT lost contact with Hayabusa2, shortly before its batteries ran out.

The MASCam images acquired during descent and bouncing reveal a surface covered with rocks and boulders of different lithologies. Four types of rock have been identified in ONC images (3). Two of these types are readily identified in MASCam images (Fig. 3): dark and rough (type 1) and bright and smooth (type 2). Type 3 rocks (bright and mottled) were not observed. In the highest resolution MASCam images, the surfaces of rock type 1 have a cauliflower-like appearance with a very small-scale rough and crumbly surface (Figs 1, $2 \mathrm{~A}, \mathrm{C}$ ). Type 2 rocks appear flat and possess elongated, non-dendritic fractures that divide the rock into planar slabs with smooth surfaces. MASCam observed rocks with sizes from decimeters to a few tens of meters. The distribution of both rock types is spread almost evenly over the surface in the areas imaged by MASCam. This observation is consistent with the interpretation that Ryugu originated 
either from two parent bodies after collision, break-up and re-accumulation resulting in two types of material or formed within the same parent body at different interior temperature and pressure conditions as a result of catastrophic disruption and redistribution also resulting in two types of material (3). Ryugu's bulk composition is expected to be similar to that of carbonaceous chondrites containing phyllosilicates and organics (CI or CM meteorites) $(2,3,8,9)$. Exposure of such materials to the diurnal temperature changes of $\sim 100 \mathrm{~K}$ on Ryugu $(2,10)$ may trigger thermal fatigue, leading to crack growth and potentially breakdown of consolidated material (11, 12). Measurements by the MASCOT radiometer (MARA) instrument indicates that the rock in front of the lander has a bulk porosity of at least $28 \%$ and a low tensile strength (10). Type 1 rocks, may respond to thermal fatigue by crumbling, resulting in their cauliflower appearances at small scales. Type 2 rocks may instead split in planar slabs, indicating preferential fracturing along weaker dimensions.

At the second location, MASCam imaged a small rock in front of the lander. Night-time images were acquired with illumination provided by onboard LEDs ( 6 ). Because of the small phase angle $\left(\sim 5^{\circ}\right)$, they reveal bright inclusions embedded in a dark matrix (Fig. 3). Most inclusions are smaller than one millimeter, but some are several millimeters across (5). In one location (Fig. 3, E) the areal coverage of the inclusions is about $10 \%$. The size of the inclusions is similar to those of chondrules (sub-mm range), calcium aluminum rich inclusions (CAIs), and other refractory inclusions in carbonaceous chondrite meteorites $(8,9)$. Typical carbonaceous chondrites of subtypes $\mathrm{CI} 2, \mathrm{CM}, \mathrm{CO}$ and $\mathrm{CV}$ possess refractory inclusions that appear similar to those in Ryugu's rock (5). However, CI1 meteorites are not a good analog due to their very low abundance of inclusions $(11,12)$. The destruction of inclusions in carbonaceous chondrites is thought to result from extensive aqueous alteration $(13,14)$, so their preservation on Ryugu is 
consistent with the notion that the asteroid was only moderately hydrated in the past (3). Ryugu's spectrum is similar to that of certain moderately heated CI and CM meteorites $(3,15)$. The CI2 type meteorite Tagish Lake, which did not experience extensive heating, has a very low albedo and abundant inclusions, like Ryugu $(5,16)$. Judging by their color, we can distinguish two broad types of inclusions: blue and red, although inclusions of different colors are also abundant (Fig. 4). Blue inclusions feature a moderately negative spectral slope of about $10 \%$ over the visible wavelength range. The positive spectral slope of the red inclusions is much steeper, in the $20-50 \%$ range. Their reflectance in the near-IR $(0.8 \mu \mathrm{m})$ is variable. Inclusions appear typically twice as bright as the rock matrix, although the smallest may be brighter as they are not spatially resolved. The spectral shape of unresolved inclusions is affected by the surrounding matrix to a minor degree, but because of their brightness they dominate the signal in each pixel. The inclusions in Fig. 4 B are well registered over the four color channels, and we expect no spectral artifacts as a result (5). The very flat nature of the matrix reflectance spectrum is consistent with ground-based spectra of Ryugu (17, $18,19)$ and the average ONC reflectance spectrum (3). The matrix reflectance has a slight excess at $0.63 \mu \mathrm{m}$ that is responsible for the reddish appearance of the rock in the color images (Fig 3E$\mathrm{J})$, but it is within the calibration uncertainty. The visible colors of the inclusions provide compositional clues, but their interpretation is limited by the lack spectral data $>1 \mu \mathrm{m}$ wavelength, where most diagnostic bands for mineralogy are located. However, we may infer 20 the composition by comparing the Ryugu inclusions to those in carbonaceous chondrites. For example, the decreasing reflectance between 0.5 and $0.8 \mu \mathrm{m}$ of the bright chondrules of the Murchison meteorite is due to olivine $(9,20)$. The similar drop in reflectance towards $0.8 \mu \mathrm{m}$ displayed by some Ryugu rock inclusions could also be due to olivine. The origin of the orange 
color of some of Murchison's larger inclusions is not known (20). The MASCam observation of abundant multi-color, millimeter-sized inclusions suggest that C-type asteroids are linked to carbonaceous chondrites.

The clearly visible inclusions suggest that the rock in front of MASCOT is not covered by microscopic (sub-mm) particles, which the MARA results confirm (10). MASCam observed no deposits of fine material during the descent. Such deposits were also absent from the surface of Itokawa (21), another rubble pile asteroid, whereas they are thought to be present on the surface of Eros (22). We expect dust to be formed continuously on the surface of Ryugu through exposure to the space environment $(23,24,25)$. However, all boulder appears clean, and therefore dust particles have likely been removed, lost either to space or the porous interior (2). The fate of fine particles is determined by a balance of forces. Cohesive forces are thought to dominate over electrostatic, gravitational, and solar radiation pressure forces for particles up to a decimeter in size, for a body as small as Ryugu (26). Although the cohesive barrier may be overcome by means of electrostatic levitation (25), the physical conditions leading to this process on small bodies are not well understood. On Ryugu, larger particles $(>100 \mu \mathrm{m})$ are more likely to be removed from the surface than finer dust (27). Alternatively, cohesion may be overcome by means of physical force, such as micrometeorite impact, seismic shaking, boulder migration and collision, drag by sublimation of volatiles, and/or thermal fracturing (25). The absence of fine deposits on the surface of small rubble piles like Ryugu and Itokawa implies the existence of an efficient removal mechanism for small particles. Gravel-sized particles may collect into the interior under the influence of gravity, but the absence of dust is not easily explained because of the strong cohesive forces involved. 


\section{References}

1. S. Watanabe et al., Space Sci Rev 208, 3-16 (2017).

2. S. Watanabe et al., Science 364, 268-272, 10.1126/science.aav8032 (2019).

3. S. Sugita et al. Science 364, 10.1126/science.aaw0422 (2019).

4. T.M. Ho et al., Space Sci Rev 208, 339-374 (2017).

5. Materials and methods are available as supplementary materials

6. R. Jaumann et al., Space Sci Rev 208, 375-400 (2017).

7. Kameda et al., Space Sci Rev 208, 17-31 (2017).

8. E.A. Cloutis et al., Icarus 212, 180-209 (2011).

9. E.A. Cloutis et al., Icarus 216, 309-346 (2011).

10. M. Grott et al., NatureAstronomie, doi.org/10.1038/s41550-019-0832-x (2019).

11. M. Delbo et al., Nature 508, 233-236 (2014).

12. J.L. Molaro et al., Icarus 294, 247-261 (2017).

13. H.Y. McSween, Geochim et Cosmochim Acta 43, 1761-1770 (1979).

14. K. Tomeoka \& P.R. Buseck, Geochim et Cosmochim Acta 49, 2149-2163 (1985).

15. K. Kitazato et al., Science 10.1126/science.aav7432 (2019).

16. T. Hiroi et al., Science 293, 2234-2236 (2001).

17. D. Lazzaro et al., Astron Astrophys 549, L2 (2013).

18. N.A. Moskovitz et al., Icarus 224, 24-31 (2013). 
19. D. Perna et al., Astron Astrophys 599, L1 (2017).

20. R.O. Green et al., 47th LPSC 2154 (2015).

21. H. Yano et al., Science 312, 1350-1353 (2006).

22. M.S. Robinson et al., Nature 413, 396-400 (2001).

23. N. Asada, J Geophys Res Solid Earth 90, 12445-12453 (1985).

24. G.J. Flynn \& D.D. Durda, Planet Space Sci 52, 1129-1140 (2004).

25. D. Jewitt, Astron. J. 143, 1-14 (2012).

26. D.J. Scheeres et al., Icarus 210, 968-984 (2010).

27. C.M. Hartzell \& D.J. Scheeres, Planet Space Sci 59, 1758-1768 (2011).

28. D. Hercik, et al., Space Science Review 208, 433-449 (2017)

29. Jaumann, R. et al., Planet. Space Sci., 55, 928-952 (2007)

30. Gwinner, K. et al., Photogramm. Eng. Remote Sens., 75, 9, 1127-1142 (2009)

31. Preusker, F. et al., Planet. Space Sci. 59, 1910-1917 (2011)

32. Raymond, C.A. et al., Space Sci. Rev. 163, 487-510, DOI 10.1007/s11214-011-9863 (2011)

33. K. Fredriksson, J.F. Kerridge, Meteoritics 23, 35-44, (1988).

34. A.J. Brearley, et al., Planetary Materials 36, Reviews in Mineralogy, (1998).

35. M. E. Zolensky et al., Meteorit Planet Sci 37, 737-761 (2002).

36. L.H. Fuchs, et al., Smithsonian Contributions to the Earth Sciences 1-39 (1973).

37. E.A. Cloutis, et al., Icarus 220, 466-486 (2012). 
38. H.Y. McSween, Geochim et Cosmochim Acta 41, 477-491 (1977).

39. J.I. Simon, et al., Earth Planet Sci Lett 494, 69-82, (2018).

Acknowledgments: The MASCOT lander on the Hayabusa2 Mission of JAXA is a DLR/CNES cooperation. MASCam was developed and built under the leadership of the DLR Institute of Planetary Research with contracted contributions of Astrium GmbH, and is operated by the DLR Institute of Planetary Research in Berlin in cooperation with the DLR Institute of Space Systems in Bremen and DLR-MUSC in Cologne. This study was supported by JSPS International Planetary Network. The authors thank H. Sadada, M. Yamada, E. Tatsumi, N. Sakatani, C. Honda, and K. Ogawa M. Hayakawa, H. Suzuki, Y. Cho, and M. Matsuoka for Hayabusa2 ONC development and science observations. We cordially thank Mike Zolensky for providing Figure S5. The Hayabusa2 Mission is operated by JAXA. Funding: The team acknowledges funding by DLR and CNES. Author contributions: R.J. coordinated and wrote the paper, R.J., N.S., S.E.S., A.K., F.T., K.D.M., and T.R. contributed to camera development, operations and data processing. R.J., N.S., S.E., F.W., F.P., F.S., K.A.O., S.K., H.J., S.E.S., S.W., M.Y., S.S. and H.R. contributed to the reconstruction of the trajectory, stereo processing, MASCOT location, comparison with ONC data and Hayabusa operations. R.J., N.S., K.A.O., K.K., R.P., J.B.V., R.W., S.S. and P.M., contributed to the geomorphological analysis and discussion of Ryugu's origin. R.J., S.E.S., K.S., N.S., S.M., J.P.B., M.G., K.A.O., and A.H., contributed to the spectral and photometric analysis. R.J., T.M.H., S.U., C.K., J.B., A.M. F.W., J.R., J.T.G., L.L., Y.T., T.O., T.Y., Y.M., and T.S. contributed to the MASCOT development, landing site selection and on surface operations. Competing interests: There are no competing interests to declare. Data and materials availability: All data is available in the main text and the supplementary materials. All images and input data used in this paper and supplementary material are available 
at http://europlanet.dlr.de/MASCAM at least until they become available at the long term archive of PDS at https://pdssbn.astro.umd.edu/. Additional data from the mission will be delivered to the DARTS archive at https://www.darts.isas.jaxa.jp/planet/project/hayabusa2/, and higher-level data products will be available in the Small Bodies Node of the Planetary Data System https://pds-smallbodies.astro.umd.edu/ one year after landing on the asteroid.

\section{Figures}



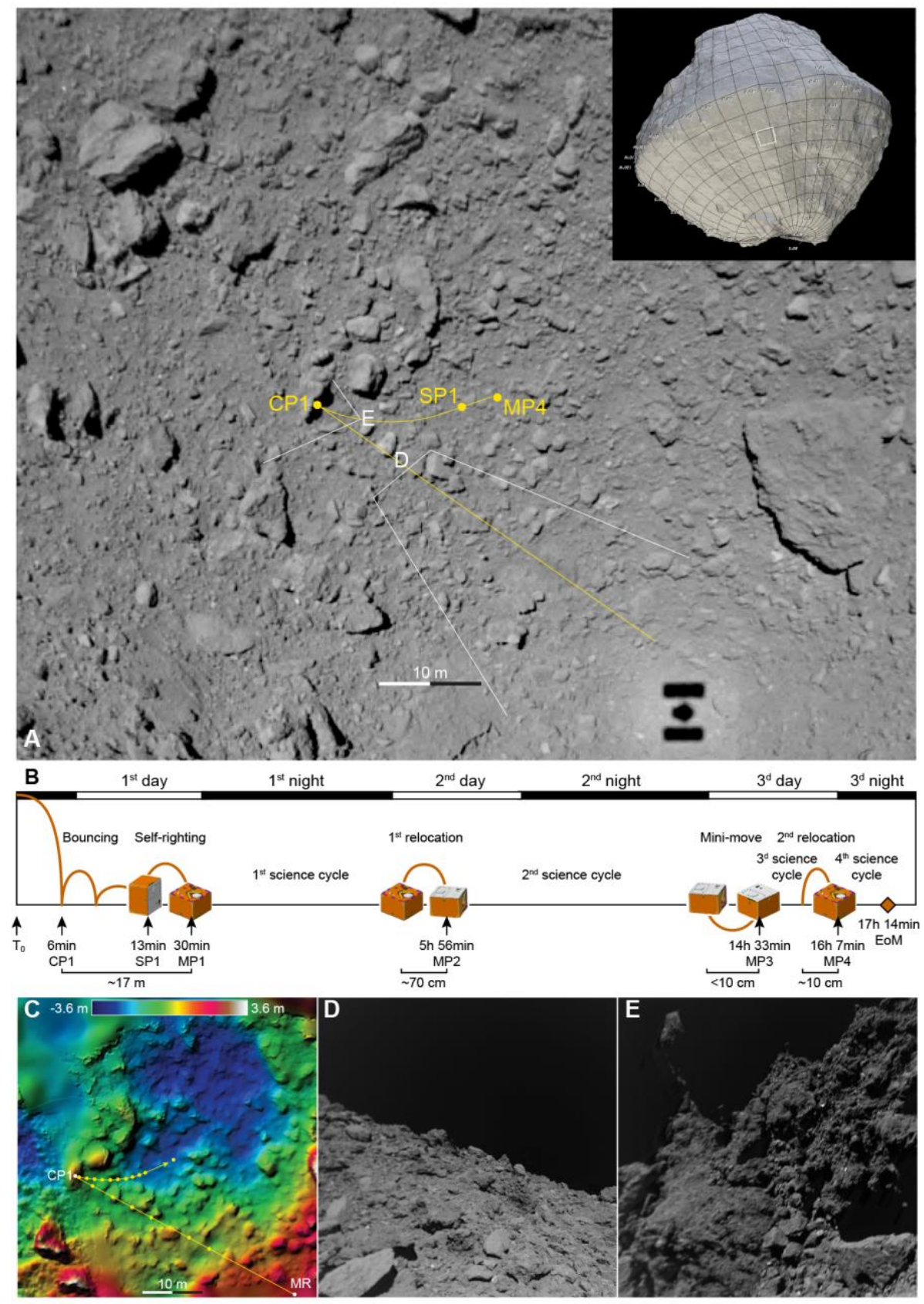

Fig. 1. Reconstructed MASCOT trajectory. (A) Hayabusa2/ONC context image, with its location on Ryugu shown in the inset. The MASCOT descent and bouncing trajectory is indicated in yellow. The MASCam field of view for two descent images shown below (d, e) are indicated by the white lines. The first contact point was CP1. (B) Schematic MASCOT mission timeline, starting from release at $T_{0}$. After bouncing, MASCOT reached SP1 (Surface Position 1) 
and acquired data in several measurement positions (MP1-4) until End of Mission (EoM) at MP4. (C) A $50 \mathrm{~m} \times 50 \mathrm{~m}$ digital terrain model of the landing area with color-coded height (defined in a local topographic coordinate frame, relative to the local tangential plane) $(3,5)$. MR is the MASCOT release point. Yellow dots indicate MASCOT positions observed in ONC images. (D) Image taken during descent, (E) image of the boulders at CP1 (scale changes with distance; the boulder in $\mathrm{D}$ lower left is about $1 \mathrm{~m}$ ). 
Late Morning

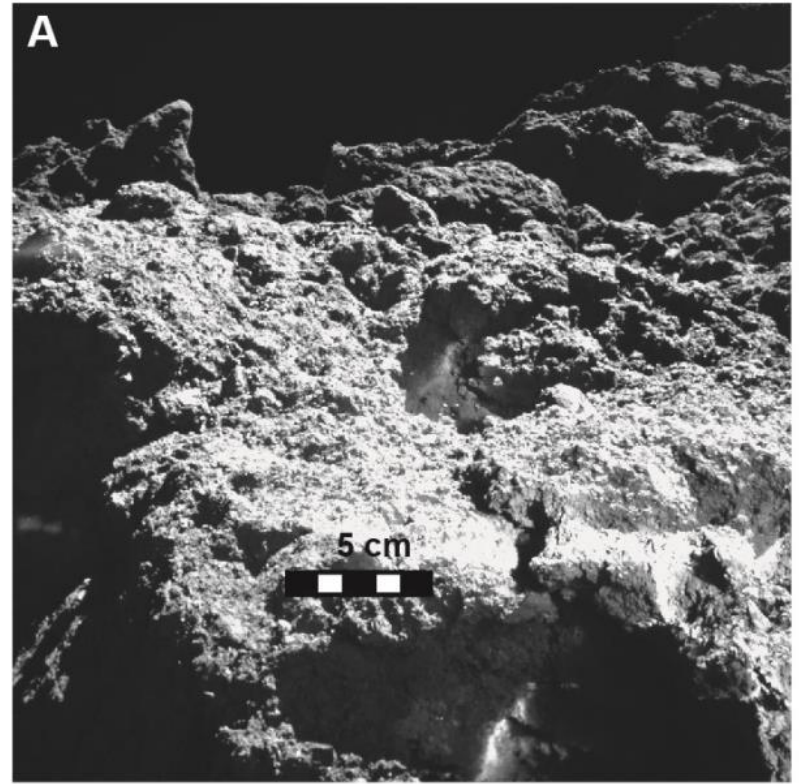

F1086982940_602_00936_n1

UTC: 2018-10-03T08:28:43.644

Local Time: 09:21:48

Noon

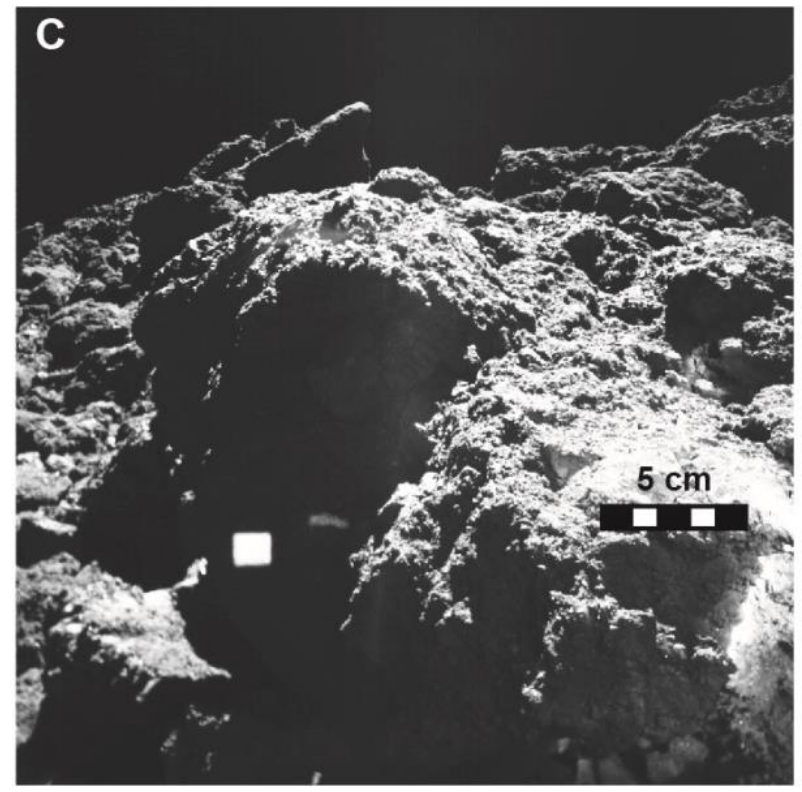

F1087923692_804_00468_r1

UTC: 2018-10-03T16:38:39.211

Local Time: 11:03:15
Night

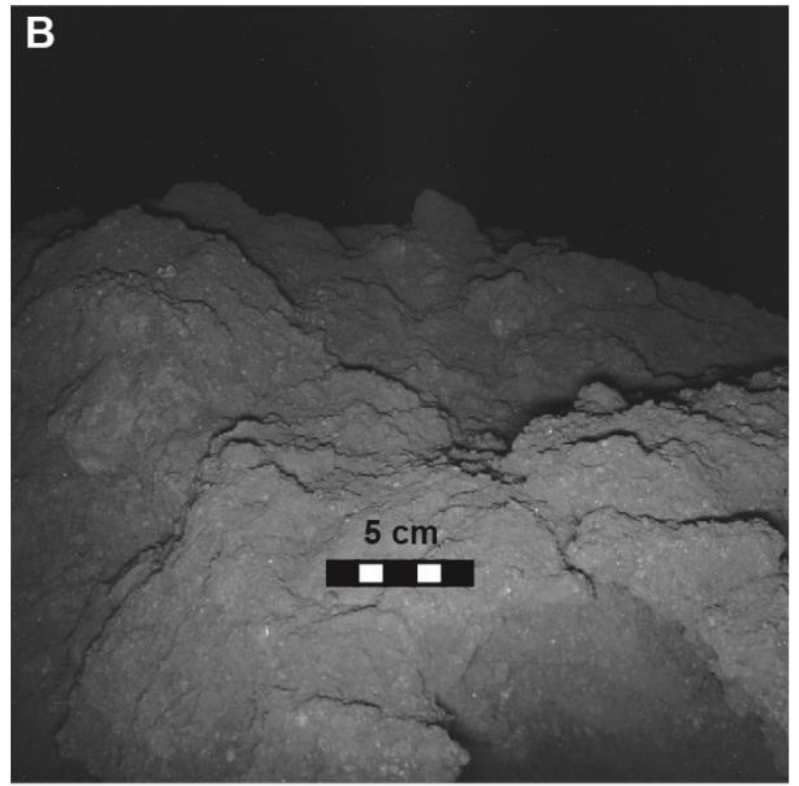

F1087378791_701_29464_r1

UTC: 2018-10-03T11:54:15.933

Local Time: 20:08:48

Late Afternoon

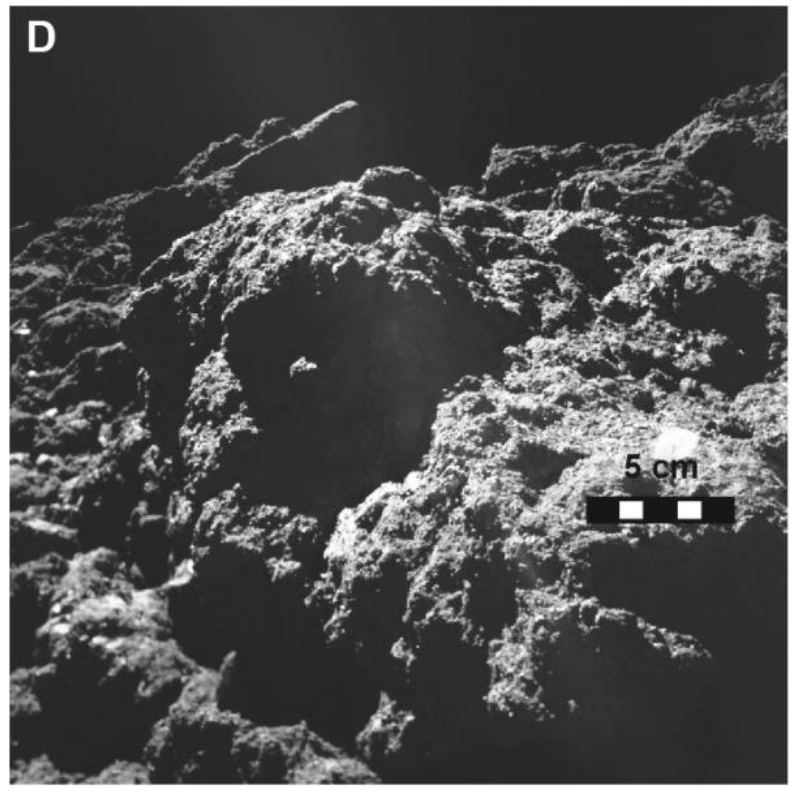

F1087998888_850_00468_r1

UTC: $2018-10-03 T 17: 17: 51: 030$

Local Time: 16:06:34

Fig. 2. Images of a type 1 rock at the second and third MASCOT locations (5). (A) Image

taken during late morning from MP2a. (B) Night time LED-illuminated image from MP2b. (C) 
Noon image from MP3a. The bright square is a reflection of sunlight of the MASCam alignment cube. (D) MASCam's last image of the surface, taken in the late afternoon from position MP3b. The bright square has moved to the right due to the change in position of the Sun. The scale is derived from a distances map in (5). The long strings of numbers under each image are the observation IDs

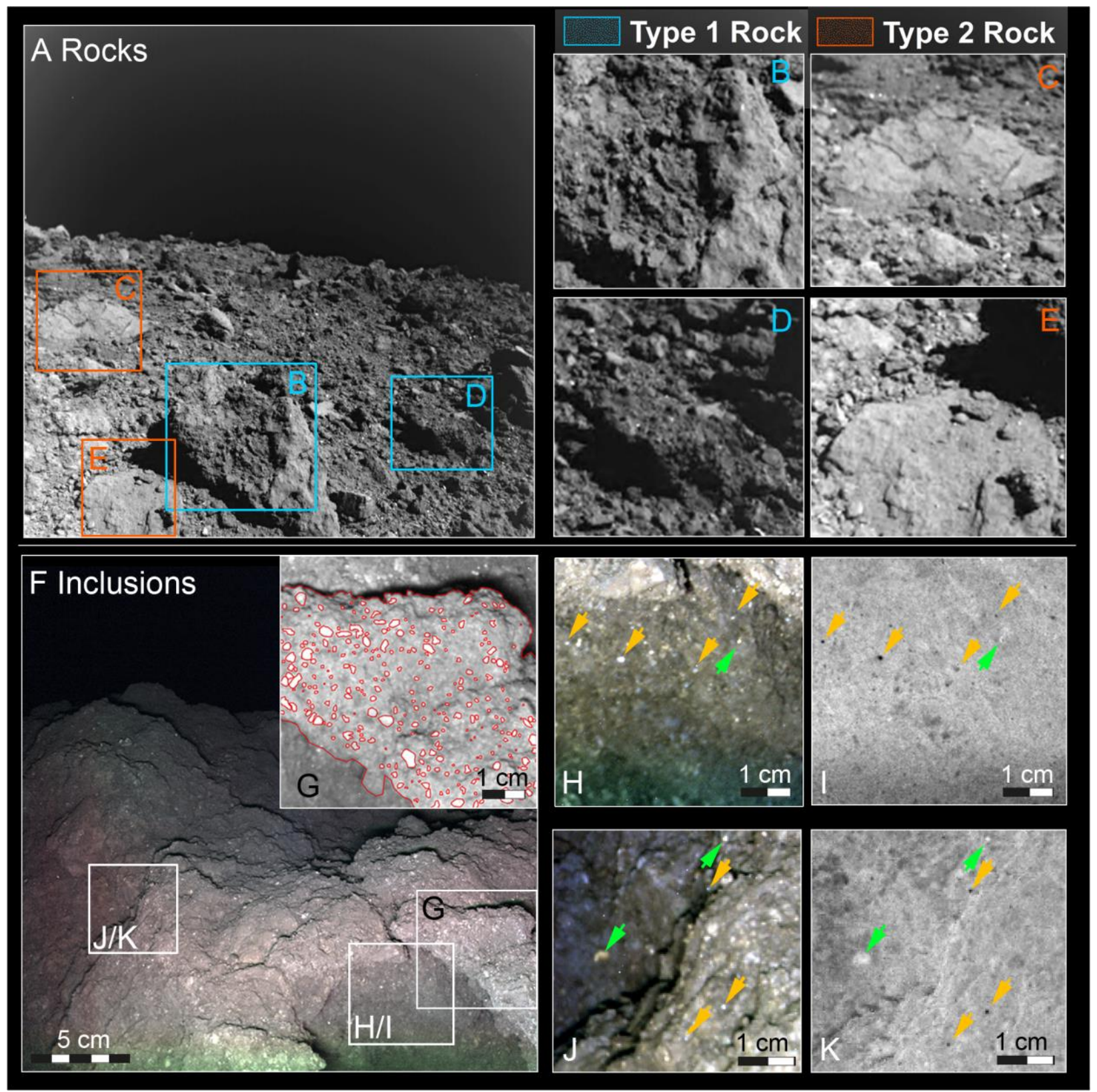


Fig. 3. Rock morphologies present on Ryugu rock. (A-E) MASCam images acquired during descent show at least two types of rocks. Rocks of type 1 appear dark with rough surfaces with a cauliflower-like, crumbly structure, whereas rocks of type 2 are smoother and brighter. (F-K) Color images $(0.465 \mu \mathrm{m}, 0.523 \mu \mathrm{m}, 0.633 \mu \mathrm{m})$ of a type 1 rock taken during the second night reveal bright inclusions. We estimated the distance to the area in $(\mathrm{G})$ as $\sim 25 \mathrm{~cm}(5)$, from which we estimate the size of the inclusions. Zoomed images of two areas $(\mathrm{H}, \mathrm{J})$ shows many inclusions are either bluish (dark, orange arrows) or reddish (bright, green arrows) in the blue $(0.47 \mu \mathrm{m})$ / IR $(0.81 \mu \mathrm{m})$ ratio images $(\mathrm{I}, \mathrm{K}) .(\mathrm{G})$ shows the size and coverage of inclusions (red contours)
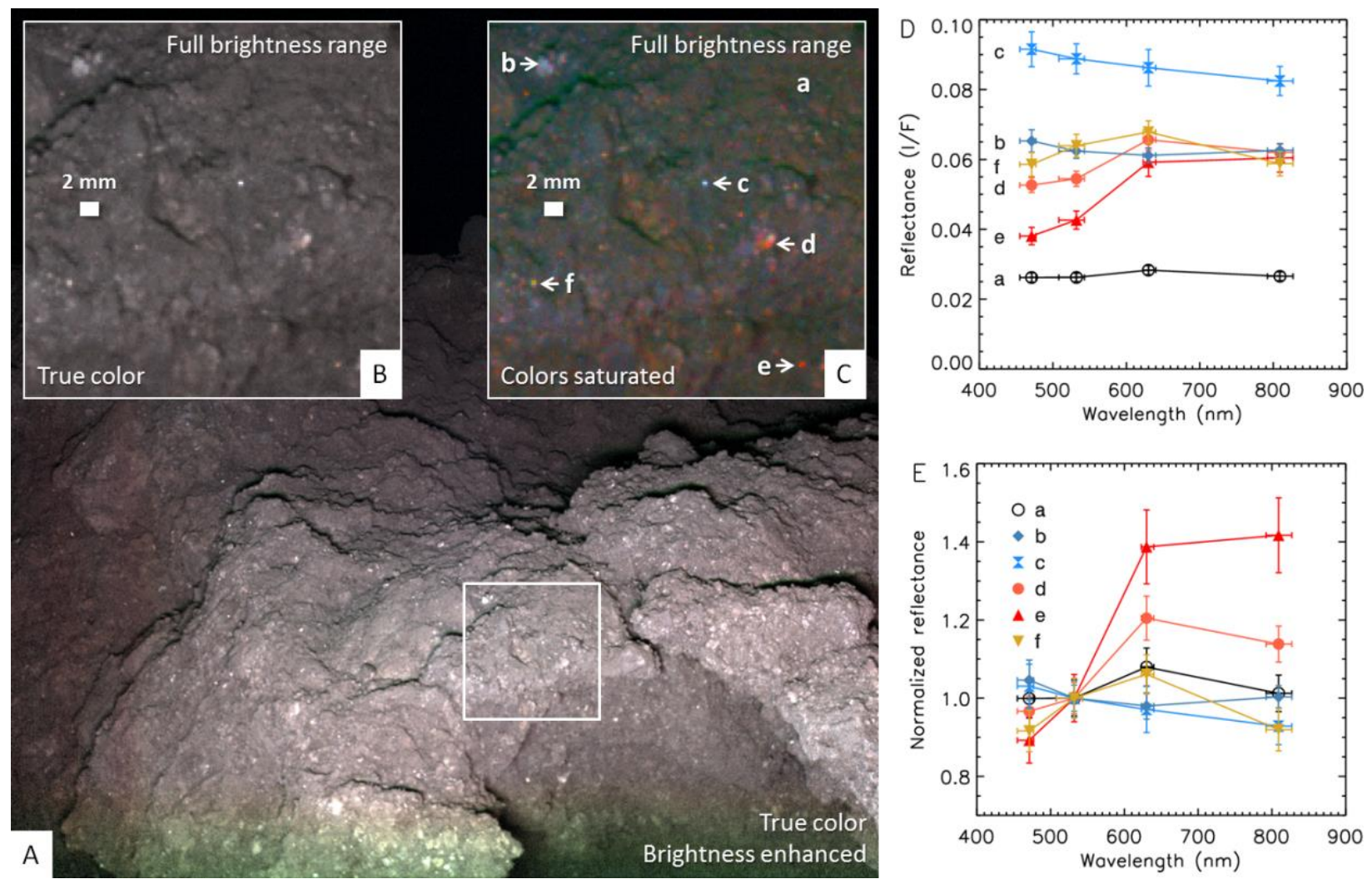
Fig. 4. Spectral variations in rock type 1. These MASCam images were taken in LED light during night. In the image center, the rock appears gray with a reddish tint, and many small, bright inclusions are visible. The insets enlarge an area in the center at the full brightness range of the LEDs to show albedo and color variations. The brightness in the true color composite $(0.465 \mu \mathrm{m}, 0.523 \mu \mathrm{m}, 0.633 \mu \mathrm{m})$ on the left is enhanced (5) to show details of the surface. The top left inset shows the area in the white box at the full brightness range, whereas the top right inset shows the same area in saturated colors. The spectra of several bright inclusions (B-F) are compared to that of the matrix (A) in the plots on the right, both as absolute I/F reflectance (top) and reflectance normalized at $0.55 \mu \mathrm{m}$ (bottom). The spectra are averages over the last three image sets of the night.

\section{Supplementary material:}

Materials and Methods, Supplementary Text, Figures. S1-S5, Tables S1-S2, References (28-39) 


\section{Science МIAAAS}

\section{Supplementary Materials for}

5

\section{Affiliations:}

Images from the surface of asteroid Ryugu show rocks similar to carbonaceous chondrite meteorites R. Jaumann ${ }^{1,2, *}$, N. Schmitz ${ }^{1}$, T.-M. Ho ${ }^{3}$, S.E. Schröder ${ }^{1}$, K.A. Otto ${ }^{1}$, K. Stephan ${ }^{1}$, S. Elgner ${ }^{1}$, K. Krohn ${ }^{1}$, F. Preusker $^{1}$, F. Scholten ${ }^{1}$, J. Biele ${ }^{4}$, S. Ulamec ${ }^{4}$, C. Krause ${ }^{4}$, S. Sugita ${ }^{5}$, K.-D. Matz ${ }^{1}$, T. Roatsch ${ }^{1}$, R. Parekh ${ }^{1,2}$, S. Mottola $^{1}$, M. Grott ${ }^{1}$, P. Michel ${ }^{6}$, F. Trauthan ${ }^{1}$, A. Koncz ${ }^{1}$, H. Michaelis ${ }^{1}$, C. Lange ${ }^{3}$, J.T. Grundmann ${ }^{3}$, M. Maibaum $^{4}$, K. Sasaki ${ }^{3}$, F. Wolff ${ }^{7}$, J. Reill ${ }^{8}$, A. Moussi-Soffys ${ }^{9}$, L. Lorda ${ }^{9}$, W. Neumann ${ }^{1}$, J.-B. Vincent ${ }^{1}$, R. Wagner ${ }^{1}$, J.-P., Bibring ${ }^{10}$, S. Kameda ${ }^{11}$, H. Yano ${ }^{12}$, S. Watanabe ${ }^{13,12}$, M. Yoshikawa ${ }^{12}$, Y. Tsuda ${ }^{12}$, T. Okada ${ }^{12}$, T. Yoshimitsu $^{12}$, Y. Mimasu ${ }^{12}$, T. Saiki ${ }^{12}$, H. Yabuta ${ }^{14}$, H. Rauer ${ }^{1,2}$, R. Honda ${ }^{15}$, T. Morota ${ }^{16}$, Y. Yokota $^{12}$, T. Kouyama $^{17}$

${ }^{1}$ German Aerospace Center (DLR), Inst. of Planetary Research, Berlin, Germany

${ }^{2}$ Freie Univ. Berlin, Inst. of Geosciences, Berlin, Germany

${ }^{3}$ DLR, Inst. of Space Systems, Bremen, Germany

${ }^{4}$ DLR, Microgravity User Support Center, Linder Höhe, Cologne, Germany

${ }^{5}$ Dept. of Earth and Planetary Science, School of Science, University of Tokyo, Tokyo 113-0033, Japan

${ }^{6}$ Univ. Côte d'Azur, Observatoire de la Côte d'Azur, Centre National de la Recherche Scientifique, Laboratoire Lagrange, Nice, France

${ }^{7}$ DLR, Inst. of System Dynamics and Control, Oberpfaffenhofen, Germany

${ }^{8}$ DLR, Inst. of Robotics and Mechatronics, Oberpfaffenhofen, Germany

${ }^{9}$ Centre National d'études Spatiales, 18 Avenue E. Belin, Toulouse 31401, France

${ }^{10}$ Univ. de Paris Sud-Orsay, Institut d'Astrophysique Spatiale, Orsay, France

${ }^{11}$ Dept. of Physics, Rikkyo University, 3-34-1 Nishi-Ikebukuro, Toshima, Tokyo 171-8501, Japan

${ }^{12}$ Inst. of Space and Astronautical Science, Japan Aerospace Exploration Agency, Sagamihara, Japan

${ }^{13}$ Dept. of Earth and Planetary Sciences, Nagoya Univ. Furo-cho Chikusa-ku, Nagoya, Japan

${ }^{14}$ Dept. of Earth and Planetary Systems Science, Hiroshima University, Hiroshima, Japan

${ }^{15}$ Kochi University, Dept. of Information Science, Akebono, Kochi, Japan

${ }^{16}$ Univ. of Tokyo, Dept. of Earth and Planetary Science, Hongo, Bunkyo, Tokyo, Japan

${ }^{17}$ National Institute of Advanced Industrial Science and Technology, Aomi, Koto, Tokyo, Japan

This PDF file includes:

Materials and Methods

The Landing Process. Depth Map and $\underline{\text { Scaling from }} \underline{\text { MASCam Images. }}$ Supplementary Text Analysis of Inclusions. Figures S1 to S5, Tables S1 to S2, References (28-39) 


\section{Materials and Methods}

\section{The Landing Process.}

It required about $4 \pm 1.5 \mathrm{sec}$. for MASCOT to leave the Mechanical and Electrical Support System (MESS), the structural interface to the Hayabusa2 mothership. The lander underwent a free-fall which lasted 6 min towards Ryugu before experiencing the first contact (CP1) on the asteroid followed by a bouncing phase of $15 \mathrm{~min} 37 \mathrm{sec}$. until it finally came to rest at the first settlement point (SP1). During the bouncing phase, the MASCOT Magnetometer (MASMag) instrument (28) detected up to three soft impacts. At SP1 the Guidance, Navigation and Control (GNC) sensors detected a false orientation of the lander, thus a self-raising maneuver was executed to turn the lander into the correct orientation for scientific measurements. Unfortunately, this was a wrong interpretation, due to a sub-threshold signal return from the dark surface or/and a cavity to the GNC sensors detecting ground proximity, and MASCOT started its $1^{\text {st }}$ science measurement cycle (MP1) upside down with the instruments looking into the sky. The measurement sequence was interrupted immediately after a link was established with Hayabusa2 and a relocation was commanded ( $1{ }^{\text {strelocation}) ~ t o ~ c o r r e c t ~ f o r ~ t h e ~ o r i e n t a t i o n ~ e r r o r . ~ M A S C O T ~ j u m p e d ~ f o r ~} \approx 70 \mathrm{~cm}$ (Fig. 1). Once it came to rest, its GNC detected the orientation correctly and the $2^{\text {nd }}$ science measurement cycle (MP2) was initiated. After its completion and a mini-move of $5 \mathrm{~cm}$ dedicated to stereo measurements (MP3), a second change of location was commanded ( $2^{\text {nd }}$ relocation). MASCOT reached its fourth and last measurement point (MP4) at an additional distance of $10 \mathrm{~cm}$. However, at this time the anticipated operational lifetime of 16 hours was already exceeded. The remaining energy just allowed MASCOT to enter a predefined "end of life" status with priority on data transmission and only minimal scientific operations.

During the descent and bouncing, MASCam performed a low cadence imaging (1/min) for $6 \mathrm{~min}$, followed by a high cadence imaging (1 per 30s) for another $6.5 \mathrm{~min}$. In total, MASCam acquired 20 descent and bouncing images, starting at 01:59:47UTC (about $2.5 \mathrm{~min}$ after separation from Hayabusa2), and ending at 02:12:27UTC (about 6.5 min before coming to rest in SP1). In addition, before, during and after the landing of MASCOT, the Optical Navigation Cameras (ONC) of Hayabusa2 took images from distances of hundreds down to tens of meters. A stereo-photogrammetric analysis (29-32)of ONC images was used to derive precise orientation parameters (orbit position and attitude) for the landing site area and to reconstruct the descent and bouncing trajectory (Fig. 1). Based on the orientation data, together with image distortion models from star calibration, an initial digital terrain model (DTM) of the landing site (Fig. 1C) was computed. Rectified ONC image maps of the landing site were produced to aid in reconstructing the descent and bouncing trajectory. In the close-up images of ONC, MASCOT's white top radiator and/or its shadow can be detected on the ground. MASCOT was visible during descent in 7 ONC images and in another $10 \mathrm{ONC}$ images during bouncing (Fig.1C). At 02:03:14UTC, MASMag recorded a first contact (CP1) with the surface, and ONC images show this happened at the shadowed side of a boulder. After deflection by the boulder, MASCOT bounced backward $\approx 17$ $\mathrm{m}$ across the surface before coming to rest in SP1(Fig. 1). The DTM reveals that, during bouncing, MASCOT traversed downwards into a local depression. At 02:14:04 UTC, ONC acquired its last image showing MASCOT during bouncing, almost 2 minutes after the last MASCam image had been taken. ONC also imaged MASCOT at its final position (Fig. S1) and confirmed the surface coordinates of $22.31 \pm 0.05^{\circ} \mathrm{S} / 317.16 \pm 0.05^{\circ} \mathrm{E}$, which allowed us to reconstruct the entire trajectory of MASCOT from its release to its final rest.

Depth Map and $\underline{\text { Scaling from MASCam Images. }}$

A small, unintentional positional change of the MASCOT lander occurred at MP3. To differentiate between the positions before and after that movement we use the denominations MP3a (before, Fig. 2C) and MP3b (after, Fig. 2D). The movement appears to have been a mainly lateral repositioning of the lander to its right, effectively creating MASCam stereo image pairs of the landscape in front of the camera. Although the baseline and the stereo angle are very small and therefore not well suited for stereo-photogrammetric analysis, we could still use the disparities created by the slightly different viewing geometries. With an increasing distance of the objects to the image plane, the disparities decrease linearly allowing the differentiation between foreground and background objects. At this point we did not possess any knowledge about the sizes of the imaged objects nor the length of the stereo base. Therefore, no absolute values for the distances to the image plane could be given, only relations between individual regions. 
Fortunately, reflections from the MASCOT structure are visible in the MASCam image. These include the rectangular reflection caused by the alignment cube (Fig. 2C \& D), a metallic cuboid at MASCOT's front used during the assembly of the lander for optical alignment. The reflection appears to be horizontally undistorted in the MP3a images, thus having the same width of $15 \mathrm{~mm}$ as the alignment cube's front side. With this information, we determined a distance of $33 \mathrm{~cm}$ between the reflection on the rock wall and the camera. The relative distances found previously were then scaled accordingly leading to a rough depth map of the area (Fig. S2). We also derived a base length of $\sim 5 \mathrm{~mm}$ between the positions of MP3a and MP3b. Due to different lighting conditions, the feature matching results are quite noisy and unreliable for background objects. Nevertheless, these estimates determine the sizes of foreground objects.

\section{Supplementary Text}

\section{Analysis of Inclusions.}

MASCam was equipped with an array of LEDs in four different colors (blue $(0.465 \mu \mathrm{m})$, green $(0.523 \mu \mathrm{m})$, red $(0.633 \mu \mathrm{m})$, and near-IR $(0.812 \mu \mathrm{m}))$ to illuminate the surface in front of the lander at night (6). The last sets of images have the highest signal-to-noise ratio as the detector temperature had dropped during the night. During the first night on the asteroid, the camera was peering into empty space, but during the second night the lander was in the correct orientation to closely observe the surface. Despite applying a correction derived from pre-launch laboratory exposures of a uniform surface, several color artifacts remain unaffected, such as the green strip at the bottom of the frame and the reddish vertical stripes at left (Fig. 3 II in report). The color balance should be most accurate to $10 \%$ in the middle of the frame. In the image center, the rock appears gray with a reddish tint, and many small, predominantly bright inclusions are visible. The inclusions are only revealed due to small phase angle of the reflected LED light $\left(<5^{\circ}\right)$, and are not evident in images acquired during the asteroid day.

The MASCam optics display minor distortion with a slight dependence on the wavelength of light. Normally, one would apply a wavelength dependent distortion correction to images acquired with LEDs of different colors to construct a properly registered color composite. We characterized the wavelength dependence of the distortion in the laboratory before launch by imaging a checkerboard target (6). However, the wavelength dependence is so minor, that our attempts to correct for it actually introduced wavelength dependent artifacts. Therefore, we performed the color analysis on images that were not corrected for distortion. Moreover, Fig. 4B \& C are located in the center of the image, where the wavelength dependent distortion should be the smallest. To illustrate the accuracy of the color registration in the inset in Fig. 4B, we enlarge the area around inclusion e in Fig. S4, preserving the shape of the original pixels. The brightest pixel is at the same location in all four images, with no perceptible color fringing around it. The apparent size of this inclusion is the same as that of the point spread function, which implies that inclusion e is probably not fully resolved. However, the accurate registration and lack of color fringing demonstrates that the inclusion is present at approximately the same location within the same pixel in all four images.

To estimate the percentage of inclusions, we manually mapped the bright inclusions in a slab of rock in front of MASCam using the night time image, illuminated by the red LED (Fig. 3). A total of 410 bright inclusions ranging between 0.03 to $4.56 \mathrm{~mm}^{2}$ were identified assuming a constant pixel resolution of $0.2 \mathrm{~mm}$ at this site. This corresponds to an area of $10 \%$ to be covered by bright inclusions. CI, CM and CO meteorites include similar sized refractory materials as the rock observed on Ryugu. Table S2 shows a comparison of inclusions in meteorites with respect to albedo, inclusion abundance and size. Using the Delesse principle (33), which states that the areal density equals the volume density, these numbers are comparable to the areal abundance of inclusions of $10 \%$ observed for the rock on Ryugu. 
Fig. S1.
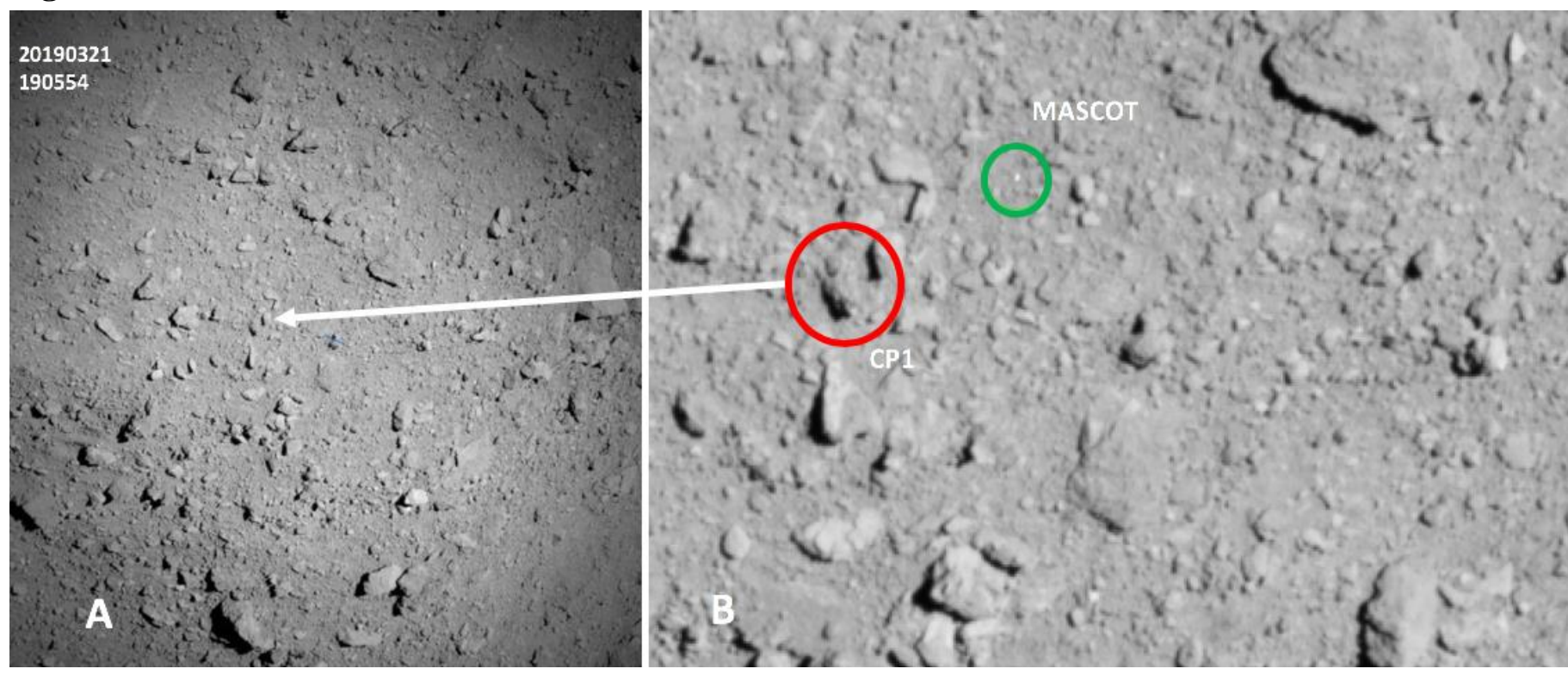

Final position of MASCOT. (A) Hayabusa 2 ONC image 20190321_190554v of the MASCOT landing area. Arrow indicates the boulder at CP1. (B) Zoom-in shows boulder at CP1 (red circle) and MASCOT as small white dot in the green circle (green circle is $4 \mathrm{~m}$ in diameter) 
Fig. S2.

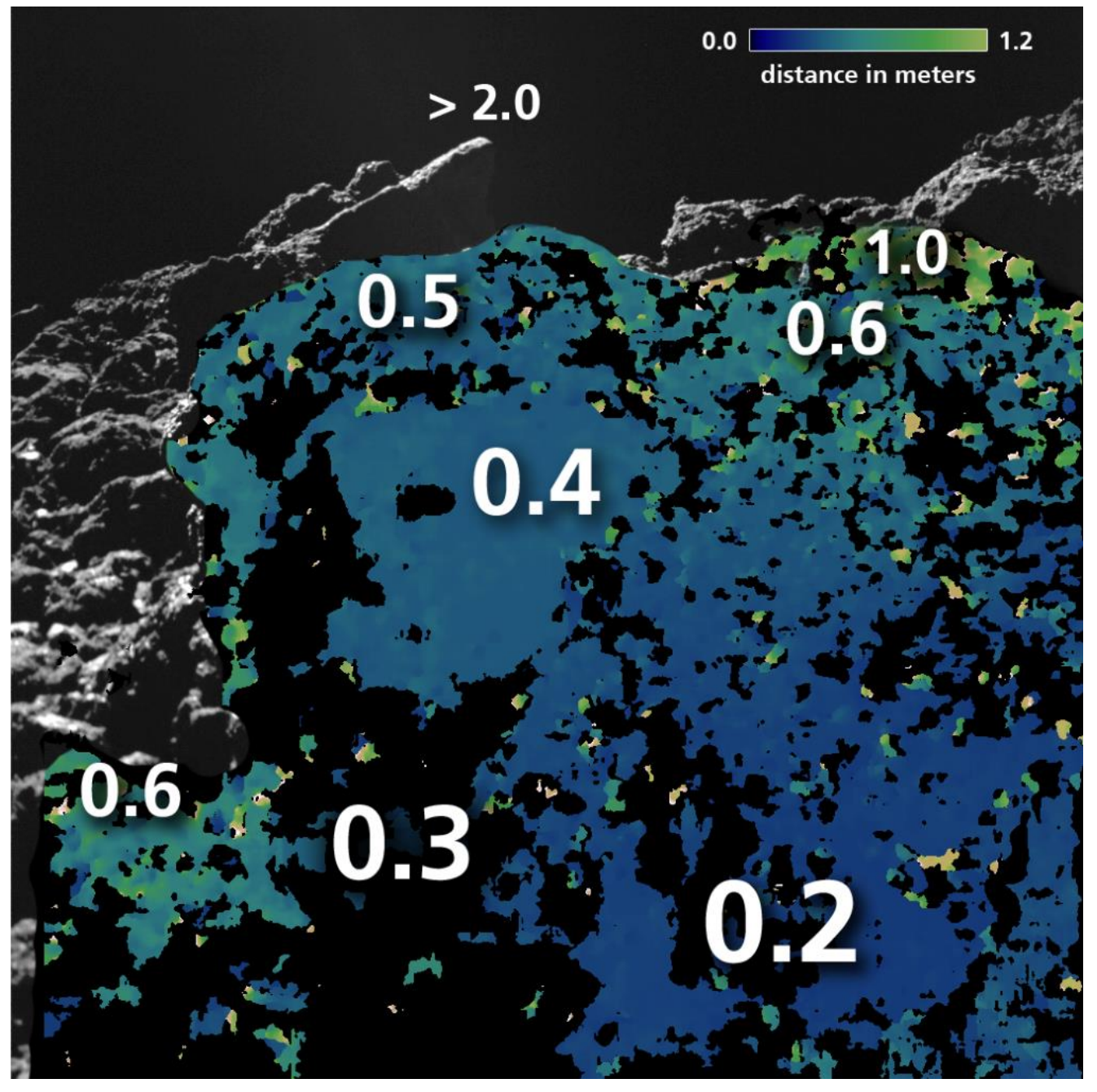

Depth map (in m) at MP2 and MP3 (see Fig. 2C \& D). Distance data are overlaid on image F1087923692_804_00468_r1. 
Fig. S3.

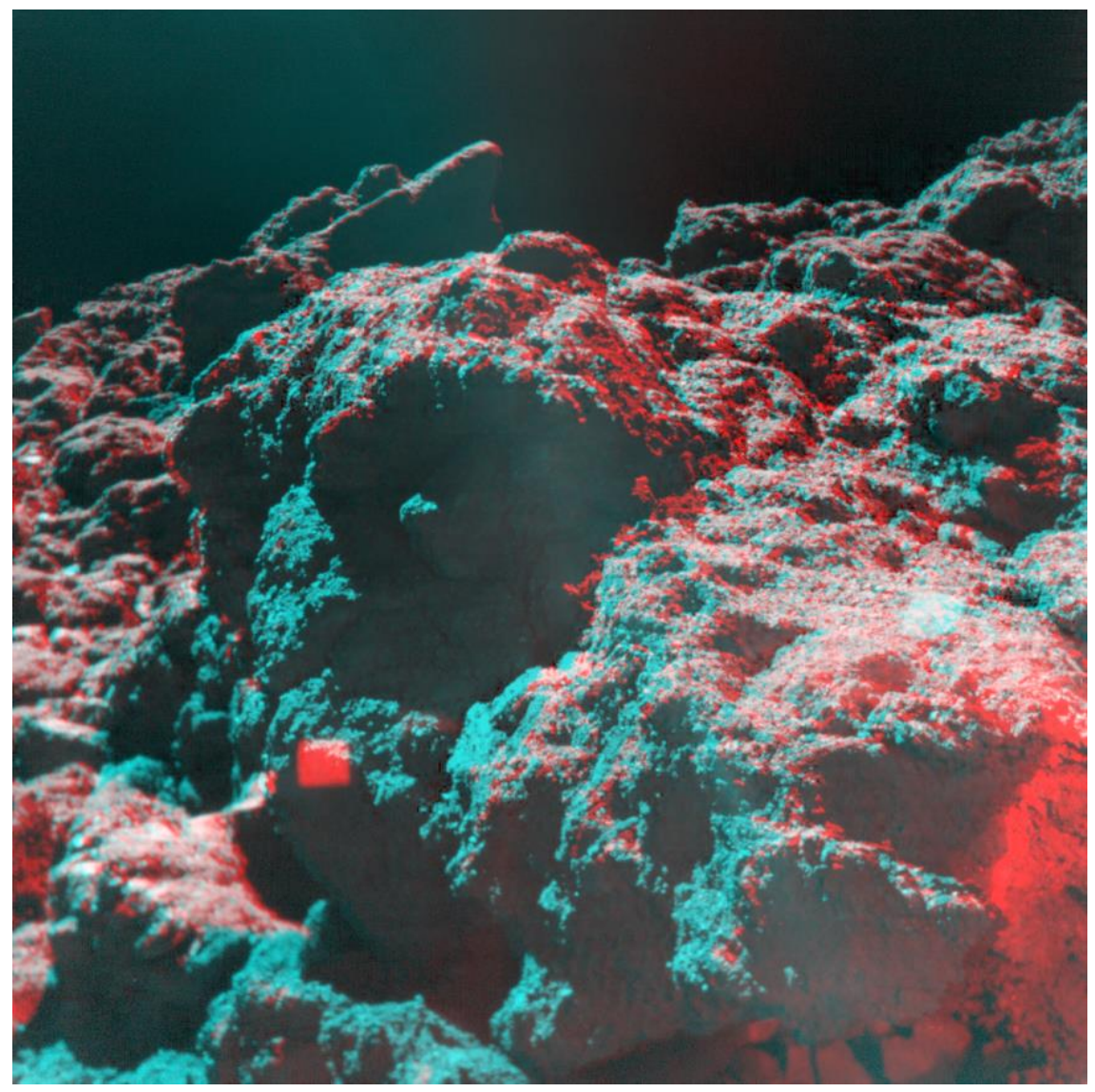

Red-cyan anaglyph image of type 1 rock at MP2 and MP3. 
AlAAAS

Fig. S4.

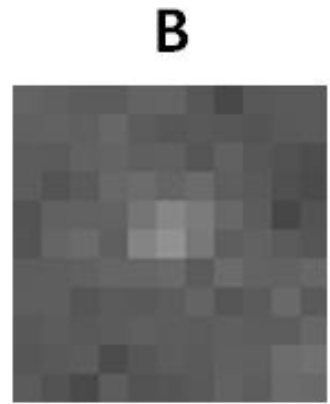

G

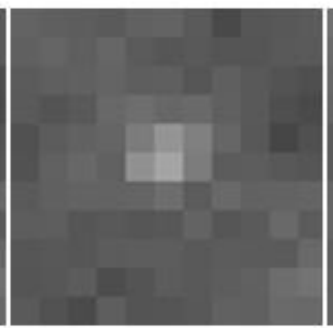

$\mathbf{R}$

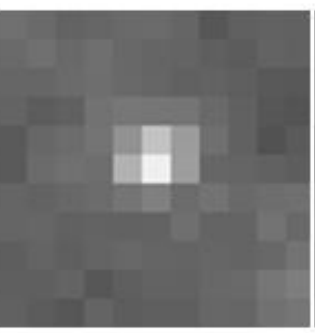

IR

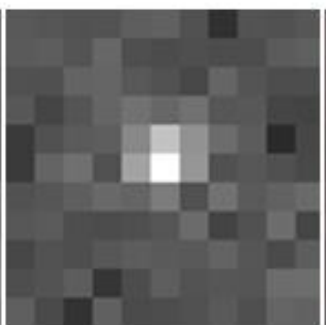

RGB

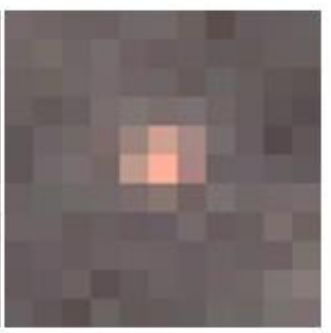

Resolution and color of inclusions. Inclusion e in Fig. 4C in calibrated images of the four different LED colors (blue, green, red, near-IR), shown on the same brightness scale (black is zero). The rightmost image is an (unsaturated) color composite of the blue, green, and red images. The pixel with the largest signal is found at the same location for all colors. 
Fig. S5.

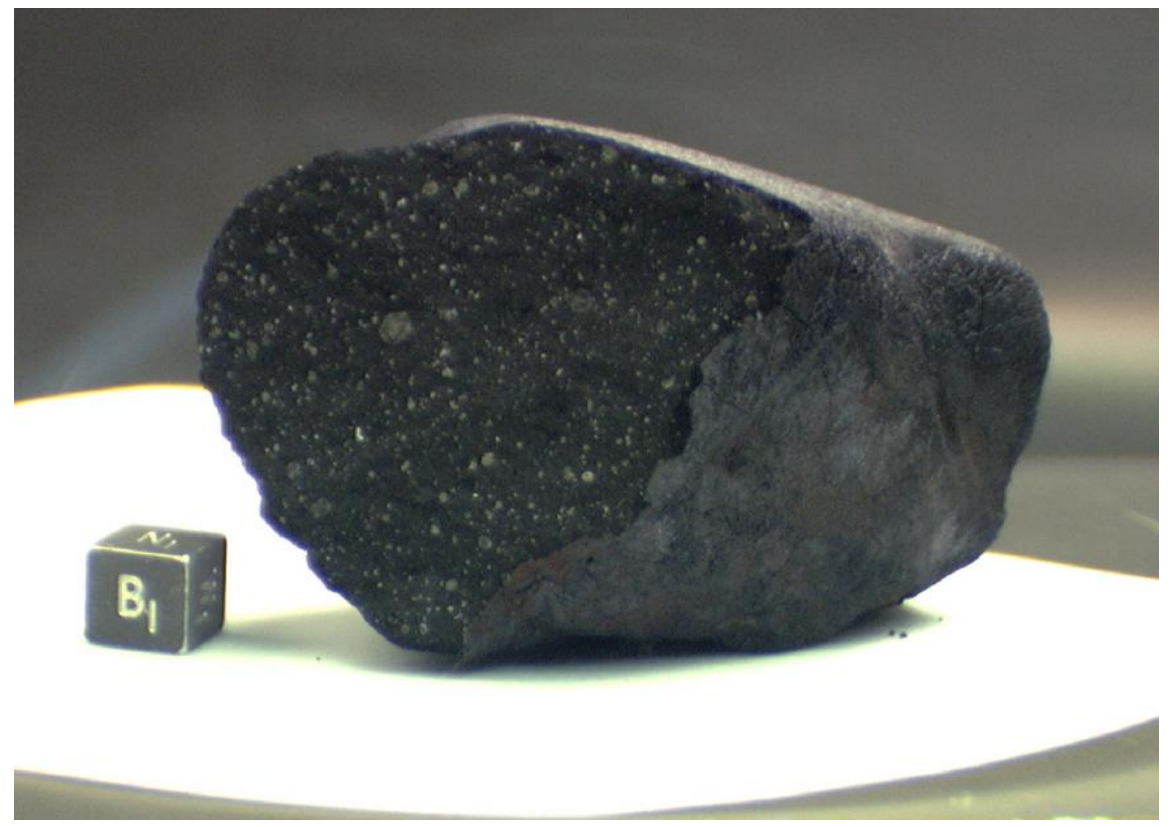

Inclusions in CI-Tagish-Lake meteorite. (Credit: by Mike Zolensky) 
Table S1.

Landing timeline. The table summarizes the main events from separation until the end of the MASCOT operations on asteroid Ryugu as well as the day-night cycles at the measurement points (see also Fig.1)

\begin{tabular}{|c|c|}
\hline Time [UTC] & Event \\
\hline $01: 57: 20$ & $\begin{array}{l}\text { MASCOT was released from Hayabusa } 2 \text { with a velocity of } \sim 4 \mathrm{~cm} / \mathrm{sec} \text {. at a } \\
\text { height of } 41 \mathrm{~m} \text { (in radial direction) above Ryugu. Afterwards it underwent a } \\
\text { free-fall towards the asteroid. }\end{array}$ \\
\hline $02: 03: 14$ & $\begin{array}{l}\text { The first impact of MASCOT with Ryugu (CP1) followed by a bouncing } \\
\text { phase. }\end{array}$ \\
\hline $02: 18: 51$ & MSC reached its first settlement point (SP1) \\
\hline $02: 34: 19$ & $\begin{array}{l}\text { MSC performed an upright and reached its first measurement point (MP1). } \\
\text { Start of the } 1^{\text {st }} \text { science cycle. }\end{array}$ \\
\hline 03:13:12 & End of $1^{\text {st }}$ day and start of $1^{\text {st }}$ night on Ryugu. \\
\hline $07: 18: 28$ & $\begin{array}{l}\text { Ground command sent to MSC to interrupt its science measurement and force } \\
\text { the lander to relocate }\end{array}$ \\
\hline $07: 51: 38$ & End of $1^{\text {st }}$ night and start of $2^{\text {nd }}$ day on Ryugu. \\
\hline $08: 27: 51$ & $\begin{array}{l}\text { MSC relocated and reached its second measurement point (MP2). The GNC } \\
\text { sensors confirmed the correct orientation of the lander and the } 2^{\text {nd }} \text { science } \\
\text { cycle was started. }\end{array}$ \\
\hline 10:50:01 & End of $2^{\text {nd }}$ day and start of $2^{\text {nd }}$ night on Ryugu. \\
\hline $15: 27: 11$ & End of $2^{\text {nd }}$ night and start of $3^{\text {rd }}$ day on Ryugu. \\
\hline $16: 30: 41$ & MSC made a mini-move (to MP3) and initiated its $3^{\text {rd }}$ science cycle \\
\hline $18: 05: 41$ & $\begin{array}{l}\text { MSC performed a } 2^{\text {nd }} \text { relocation. It reached its } 4^{\text {th }} \text { measurement point (MP4) } \\
\text { and executed measurement of MASCAM and other instruments }\end{array}$ \\
\hline 18:29:11 & End of $3^{\text {rd }}$ day and start of $3^{\text {rd }}$ night on Ryugu. \\
\hline 19:04 & $\begin{array}{l}\text { End of mission: reception of last MASCOT house-keeping packets (final link } \\
\text { break by horizon occultation). }\end{array}$ \\
\hline
\end{tabular}




\section{Table S2.}

Comparison of Ryugu with meteorites. Rock reflectance factors at $0.55 \mu \mathrm{m}$, inclusion and chondrule properties of Ryugu and five carbonaceous chondrites. All meteorites have attributes in common with Ryugu's rock. While differences in albedo can be introduced by measuring techniques and grain sizes, the number of refractory inclusions in the meteorites is a strong indication for similarities. They suggest that meteorites, such as CMs and Tagish Lake, are the best analogues for Ryugu.

\begin{tabular}{|c|c|c|c|c|c|}
\hline Meteorite & $\begin{array}{l}\text { Reflectance } \\
\text { factor at } 0.55 \\
\mu \mathrm{m}\end{array}$ & $\begin{array}{l}\text { Refractory } \\
\text { inclusion } \\
\text { abundance } \\
\text { (vol\%) }\end{array}$ & $\begin{array}{l}\text { Inclusion size } \\
(\mathrm{mm})\end{array}$ & $\begin{array}{l}\text { Chondrule } \\
\text { inclusion } \\
\text { abundance } \\
\text { (vol\%) } \\
\end{array}$ & $\begin{array}{l}\text { Chondrule } \\
\text { mean } \\
\text { diameter } \\
(\mathrm{mm})\end{array}$ \\
\hline Cl1 (Orgueil) & $0.063(3)$ & $<<1(8)$ & $0.1-3^{*}(33)$ & $<<1(34)$ & - \\
\hline $\begin{array}{ll}\mathrm{Cl} 2 & \text { (Tagish } \\
\text { Lake) } & \end{array}$ & $0.02(16)$ & $6 * *$ & $\begin{array}{l}0.1-0.5 \quad(35) \\
<0.6^{* *}\end{array}$ & $\sim 1$ (35) & $0.25-1(35)$ \\
\hline $\begin{array}{l}\text { CM2 } \\
\text { (Murchison) }\end{array}$ & 0.065 (3) & $5(34)$ & $0.5-4.5(36)$ & $20(34)$ & $0.3(34)$ \\
\hline CO3 (Ornans) & $0.10-0.13(37)$ & $13(34)$ & $0.2-0.5^{*}(38)$ & $48(34)$ & $0.15(34)$ \\
\hline CV4 (Allende) & $0.086(3)$ & $10(34)$ & $0.29 \pm 0.38(39)$ & $45(34)$ & $1.0(34)$ \\
\hline Ryugu & $0.02(3)$ & 10 & $0.38 \pm 0.55$ & & \\
\hline
\end{tabular}

